\title{
Performance Evaluation of Bluetooth Low Energy for High Data Rate Body Area Networks
}

\author{
José A. Afonso ${ }^{1}$, António F. Maio ${ }^{1}$, Ricardo Simoes ${ }^{2,3}$ \\ ${ }^{1}$ Centre for Microelectromechanical Systems (CMEMS-UMinho), University of Minho, Guimarães, \\ 4800-058, Portugal \\ ${ }^{2}$ Institute for Polymers and Composites (IPC/I3N), University of Minho, Guimarães, Portugal \\ ${ }^{3}$ School of Technology, Polytechnic Institute of Cávado and Ave, Barcelos, Portugal \\ ${ }^{1}$ E-mail: jose.afonso@dei.uminho.pt, Phone: +351-253510190, Fax: +351-253510189
}

\begin{abstract}
Bluetooth Low Energy (BLE) is a promising wireless network technology, in the context of body area network (BAN) applications, to provide the required quality of service (QoS) support concerning the communication between sensor nodes placed on a user's body and a personal device, such as a smartphone. Most previous BLE performance studies in the literature have focused primarily in networks with a single slave (point-to-point link) or traffic scenarios with relatively low data rate. However, many BAN sensors generate high data rate traffic, and several sensor nodes (slaves) may be actively sending data in the same BAN. Therefore, this work focuses on the evaluation of the suitability of BLE mainly under these conditions. Results show that, for the same traffic, the BLE protocol presents lower energy consumption and supports more sensor nodes than an alternative IEEE 802.15.4-based protocol. This study also identifies and characterizes some implementation constraints on the tested platforms that impose limits on the achievable performance.
\end{abstract}

Keywords: Bluetooth Low Energy, Performance Evaluation, Body Area Networks, Quality of Service.

This manuscript was published in Wireless Personal Communication.

The final publication is available at link.springer.com

http://www.springer.com/-/2/AVRPIOwWnYabzt6nUIzl 


\section{Introduction}

Body area networks (BAN) [1] allow users to be monitored continuously anytime, anywhere, while they are engaged in their daily life activities. BANs are mainly composed of wearable and/or implantable nodes and one or more wireless networks, which are used to transport the data collected from the sensor nodes. There are several parameters which can be monitored using a BAN device, such as heart rate, blood glucose level, blood pressure, body posture, electroencephalography (EEG), electrocardiography (ECG), electromyography (EMG), among others [2]. Application areas for BANs include healthcare, sports and entertainment.

Body area networks may be implemented using different architectures. In [3], the architecture of these systems is structured into three layers: intra-BAN, inter-BAN and beyond-BAN. The intra-BAN layer involves communication between multiple BAN nodes placed inside or over the user's body (e.g., implantable or wearable sensors or actuators) and a personal device (hub), which is also carried by the user. The personal device may be either a dedicated device designed specifically for this function or a general-purpose device, such as a smartphone.

The second layer (inter-BAN) provides wireless communication, either directly of passing through wireless routers, between the personal device and an access point or base station located outside the body. This layer may use wireless network technologies such as IEEE $802.11 / \mathrm{Wi}-\mathrm{Fi}$ or cellular networks, and the same network may serve multiple users. Alternatively, some systems (e.g. [4]) may provide direct communication between the BAN nodes on the body and an external access point, thus integrating the first two layers and eliminating the need for a personal device.

The third layer (beyond-BAN) aims to provide services such the remote storage and access to information collected from the users. An example is a client/server system for remote access of clinical records of patients, stored in a database, through the Internet.

Given its characteristics, Bluetooth Low Energy (BLE) seems one of the better suited network technologies, in the context of the intra-BAN layer, to provide wireless communications between the BAN nodes and the personal device. In comparison with Bluetooth, BLE provides lower energy consumption. Compared with IEEE 
802.15.4/ZigBee [5], [6], one of the advantages of BLE is that the BLE radio interface is generally available in smartphones, especially the newer models, whereas native support for IEEE 802.15.4 is practically nonexistent. The IEEE 802.15.6 BAN standard [7] does not have products available in the market yet. BLE may also be suitable, for short distances, to provide direct wireless communication between BAN nodes placed on one or more users and a nearby access point.

Bluetooth Low Energy has application in several areas, such as healthcare [8], [9] [10], entertainment and sports/fitness [11]. Omre and Keeping [8] discuss the application of BLE for medical monitoring and presents an example application focusing on blood glucose monitoring for management of diabetes. Chan et al. [10] present a BLE patch sensor device containing ECG electrodes, a microcontroller, a triaxial accelerometer and a BLE transceiver, and discuss its utilization for remote measurement of heart rate, respiration, activity and falls. Jara et al. [9] analyze the capabilities of BLE for continuous data transmission in home-care and Ambient Assisted Living (AAL) environments.

Maio and Afonso [11] describe a system that allows the monitoring of the posture of cyclists in real-time, which has influence on the performance of the athletes. Raw data are collected from sensor nodes containing accelerometers, magnetometers and gyroscopes, which are placed in the trunk and legs, and are sent to an Android smartphone using BLE, where this information is processed and the trunk and knee angles are calculated.

Lin et al. [12] discuss the application of BLE in the automotive industry, proposing the use of BLE as an intra-vehicular wireless sensor network (WSN), in order to provide alternative solutions for addressing potential issues that arise from the use of additional wiring, to help the development of vehicles with better fuel economy and performance, and to support new applications.

In [13], the authors identify BLE as one of the most promising technologies enabling the Internet of Things (IoT) [14], whereas in [15] the authors present networking solutions, based on standardization work currently being developed in the Bluetooth Special Interest Group and IETF (Internet Engineering Task Force), for connecting BLE devices to the IoT. 
Several performance parameters of BLE networks have been studied in the literature. Siekkinen et al. [16] present a study of the energy consumption of BLE devices. Results show that BLE is very energy efficient in terms of energy per bit transmitted when compared to IEEE 802.15.4/ZigBee. The authors also identify some specific limitations of the stack implementation used in the study, referring that the energy efficiency could be further improved by implementing AFH (Adaptive Frequency Hopping).

Gomez et al. [17] present an analytical model for the maximum throughput of BLE for unidirectional data communication from the master to a single slave in the presence of uncorrelated bit errors, for different values of connection interval. Simulation results are used to validate the proposed analytical model.

Mikhaylov et al. [18] discuss and compare the maximum throughput, the minimum turnaround time and the energy consumption for three protocols: BLE, IEEE 802.15.4 and SimpliciTI, based on theoretical analysis and experimental measurements. In [19], the authors evaluate the energy consumption, latency, piconet size, and throughput of BLE as a function of the connection interval and slave latency, either by means of theoretical, simulation or experimental results. According to theoretical results, the lifetime of a BLE device powered by a $230 \mathrm{mAh}$ coin cell battery ranges between 2.0 days and 14.1 years, whereas the number of simultaneous supported slaves in a BLE piconet ranges between 2 and 5,917. Both of these papers identify some limitations in the performance of the BLE network caused by implementation constraints in the BLE stack of the used CC2540 modules.

This work presents several novel contributions in comparison with the performance analyses of the BLE protocol referred above. In a scenario where sensor nodes send a single packet per connection interval, it may be possible to achieve extremely high values for the battery lifetime and the number of supported nodes when the connection interval is very high, as shown in [19]. However, in this case, the data rate generated by each individual sensor node is extremely low. In contrast, this work provides an evaluation of the energy consumption and number of supported nodes in the context of a real application, where the sampling frequency for the sensor nodes is fixed, which means that the data rate generated by the nodes is independent of the connection interval. 
Another goal of this work is to evaluate the quality of service (QoS) support of BLE for the transport of the traffic of multiple high data rate sources. For this purpose, we consider the traffic parameters from a wireless posture monitoring application [20] where each sensor node contains nine sensors that are sampled at $30 \mathrm{~Hz}$, which is equivalent to a single sensor being sampled at $270 \mathrm{~Hz}$. There are several other BAN signals that may present similar data-intensive traffic, such as ECG, EEG or EMG. As an example, the monitoring of ECG signals from patients may require a sampling frequency as high as $250 \mathrm{~Hz}$ per electrode [21].

The mandatory CSMA/CA (Carrier Sense Multiple Access/Collision Avoidance) MAC (Medium Access Control) protocol used by IEEE 802.15.4/ZigBee networks is not suitable to provide the QoS support required by the traffic of multiple high data rate sensor nodes, due to the increased probability of collisions [22]. The optional collisionfree GTS (Guaranteed Time Slot) scheme also has some drawbacks (for example, it only supports, at most, seven GTS allocations). The eLPRT (enhanced Low Power Real Time) protocol [23], which was implemented on top of the physical layer of the IEEE 802.15.4 standard, was conceived with the goal of providing better QoS support under these high load traffic scenarios. Therefore, this protocol was selected in this work for comparison with the BLE protocol.

Like previous works [18], [19], this paper provides experimental BLE results using CC2540 modules. Unlike those works, this paper also evaluates the performance of BLE networks using a smartphone as the central station, which is a typical configuration, especially in the context of BAN applications. This work also presents a more detailed evaluation of the implementation constraints of the tested platforms.

The main contributions of this paper are: a theoretical analysis of the performance of BLE with multiple sensor nodes and high data rate traffic; an experimental evaluation of the reliability of BLE with small connection intervals and multiple sensor nodes, using two different BLE platforms; and a comparison of performance, in terms of the number of supported slaves and energy consumption, between the BLE and eLPRT protocols.

The rest of the paper is organized as follows. Section 2 presents a brief overview of the BLE protocol. Section 3 presents an analysis of relevant BLE parameters, in the 
context of this work, as well as a brief analysis of the eLPRT protocol. Section 4 presents and discusses experimental and theoretical results based on the analysis performed in the previous section. Finally, Section 5 presents the conclusions.

\section{BLE Overview}

This section presents a brief overview of the characteristics of the BLE protocol [24], [19] that are more relevant in the context of this work. The reader can find more detailed descriptions of BLE in the provided references.

BLE is the main feature introduced by the Bluetooth 4.0 specification. Devices that support both the legacy BR/EDR (Basic Rate/Enhanced Data Rate) controller and the new LE (Low Energy) controller are referred to as dual-mode devices and fall into the Bluetooth Smart Ready category. Typically, smartphones and laptops are examples of dual-mode devices in a Bluetooth 4.0 system. Devices that support only LE are referred to as single-mode devices and fall into the Bluetooth Smart category. These devices usually require low power consumption, are powered using batteries and have small size.

Bluetooth Low Energy operates in the $2.4 \mathrm{GHz}$ frequency band using frequency hopping spread spectrum (FHSS). There are two types of RF channels: advertising channels and data channels. BLE provides three advertising channels, which are used to discover devices, establish connections and broadcast data, and 37 data channels, which are used for bidirectional communication between devices. The bit rate provided at the physical layer is $1 \mathrm{Mbps}$.

Similarly to Bluetooth, BLE defines two device roles at the link layer for a connection: master and slave. A network (which is called a piconet) is composed of one master and one or more slaves, and is based on a star topology.

Fig. 1 presents the data packet format at each layer of the BLE protocol stack, identifying the different fields that are relevant in the context of the evaluation performed in this paper and their respective sizes. The main goal of L2CAP (Logical Link Control and Adaptation) layer in BLE is to multiplex the data of three higher layer 
protocols: ATT (Attribute Protocol), SMP (Security Manager Protocol) and link layer control signaling, on top of a link layer connection.

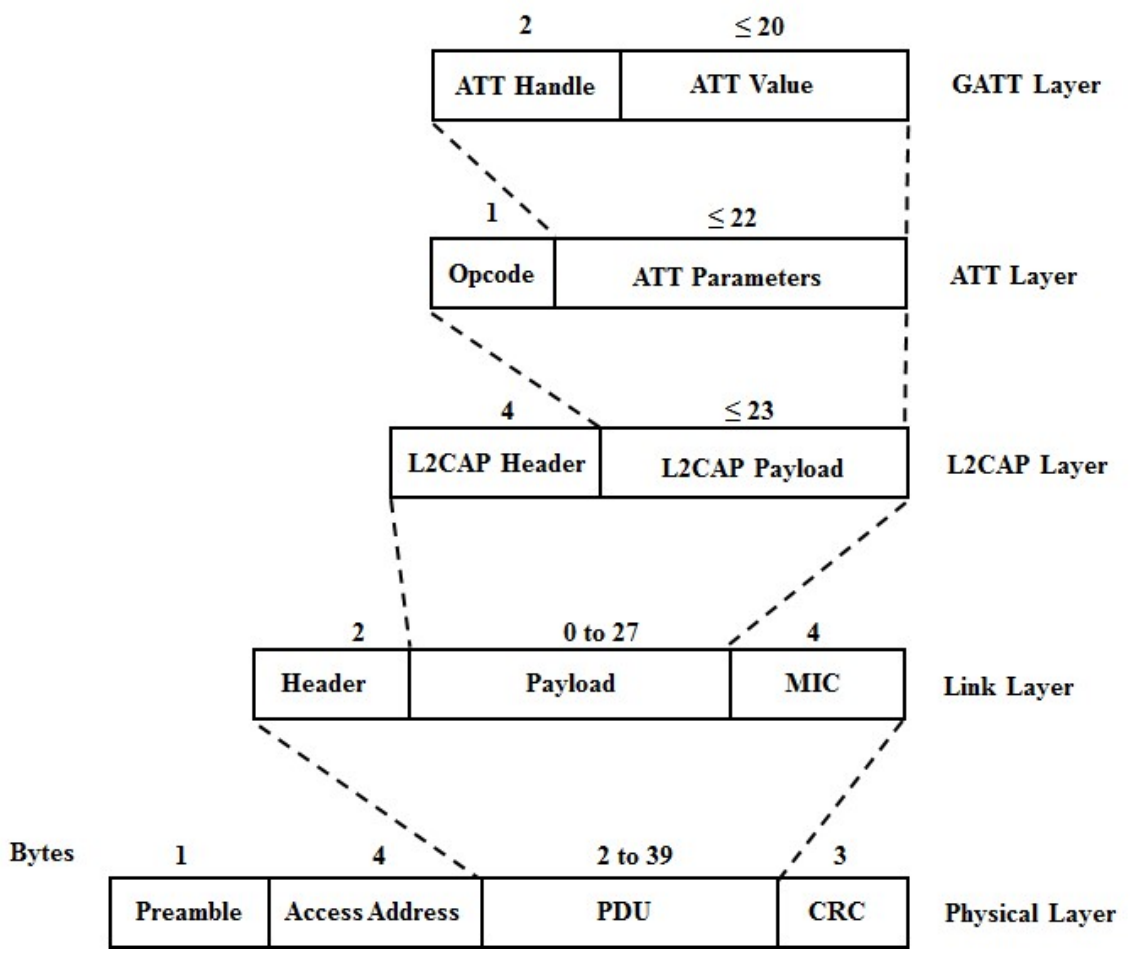

Fig. 1 BLE data packet format at different layers

The ATT defines the communication between two devices with the roles of server and client. The server maintains a set of attributes, storing information managed by the GATT protocol, which operates on top of the ATT. The client can access the server's attributes by sending requests, which originate responses from the server, or send commands to the server in order to write attribute values. A server can also send unsolicited messages to a client, containing attributes, by using either notifications or indications. The former are unconfirmed, whereas the latter require the client to send back a confirmation. The protocol evaluation performed in this work is based on the use of notifications sent from the slaves to the master.

Among the roles specified by the BLE GAP (Generic Access Profile), the role of central station is attributed to a device responsible for initiating and managing multiple connections, whereas the role of peripheral station is designed for simple devices which may only establish a single connection with the central station. Consequently, the 
central and peripheral roles require the device to support either the master or slave roles, respectively, at a given time.

\subsection{Connection Events}

After a connection between a master and a slave is established, time is divided into non-overlapping periods called connection events. Communication during a connection event is started by the master, which sends a packet to the slave. The master and the slave alternate the transmission of packets on the data channel until one of the devices does not have more data to transmit or until the connection event is over. During a connection event, all packets are transmitted using the same channel frequency. The interval between active connection events, which may also be called effective connection interval $\left(T_{e c i}\right)$, depends on two parameters: the connection interval $\left(T_{c i}\right)$ and the slave latency $\left(N_{s l}\right)$, and can be calculated using equation (1).

$$
T_{e c i}=T_{c i}\left(1+N_{s l}\right)
$$

Therefore, when the slave latency is equal to zero, the interval between active connection events is equal to the connection interval. The connection interval may range from $7.5 \mathrm{~ms}$ to $4 \mathrm{~s}$, in multiples of $1.25 \mathrm{~ms}$, whereas the slave latency may range from 0 to 499 .

Fig. 2 illustrates the packet exchange between the master and a slave for a connection event (polling scheme) where the slave latency is zero. $T_{I F S}$ is the time interval between two consecutive packets on the same channel (interframe space), and has a duration of $150 \mathrm{~ms}$.

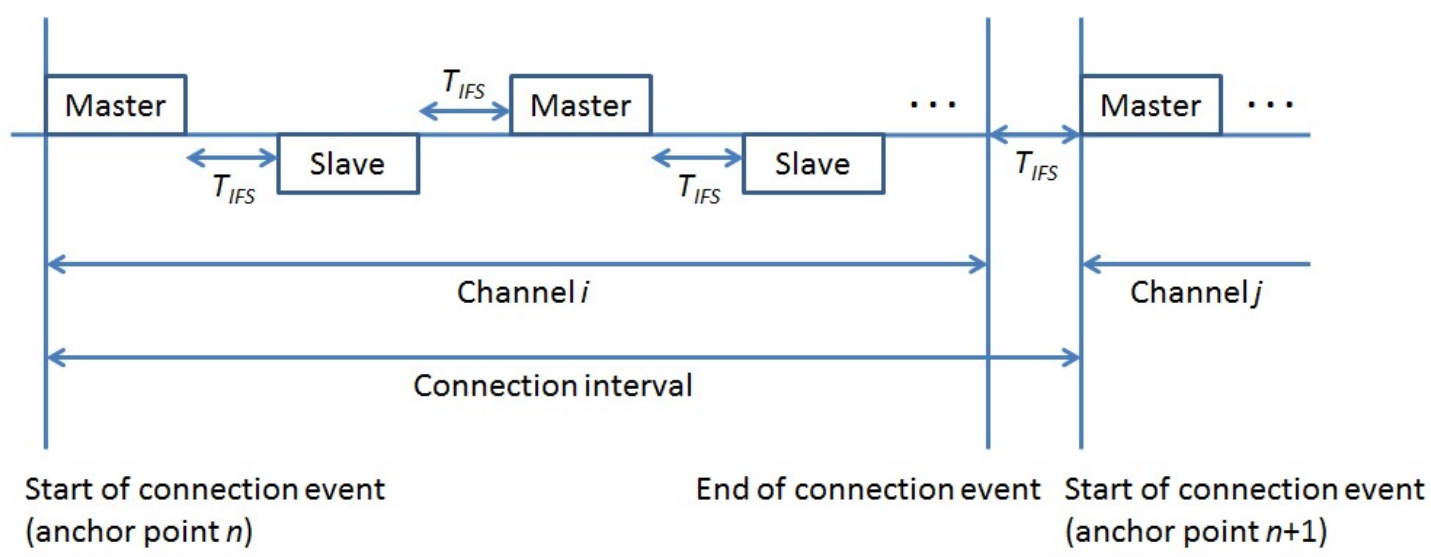


Fig. 2 Data exchange between the master and a slave in the data channel

The MD (More Data) bit present in the layer PDU (Protocol Data Unit) header indicates whether the device has more data to send. If none of the devices have enabled the MD bit in their packets, the packet from the slave terminates the connection event.

\section{Protocol Analysis}

This section presents an analysis of the BLE protocol, focusing on the protocol throughput, energy consumption and number of supported slaves. It also provides a brief analysis of the eLPRT protocol, which is used in section 4 to provide performance comparison results between these two protocols.

\subsection{Throughput}

The data transmission mode considered in this paper is the sending of notifications from an ATT server (slave) to an ATT client (master). Under this mode, the notification packets are sent by the server without the requirement of prior reception of a command, and the client also does not have to confirm the reception of the data.

Table 1 presents the transmission times for all fields (depicted in Fig. 1) of the packets exchanged between the master and the slave. These times were calculated based on the length of the fields, taking into account that the BLE bit rate is $1 \mathrm{Mbps}$.

Table 1 Detailed transmission times of the fields of the master and slave packets

\begin{tabular}{|c|c|c|c|}
\hline Acronym & Field & Bytes & Transmission Time \\
\hline$T_{p r e}$ & Preamble & 1 & $8 \mu \mathrm{s}$ \\
\hline$T_{a a}$ & Access address & 4 & $32 \mu \mathrm{s}$ \\
\hline$T_{l h}$ & Link layer header & 2 & $16 \mu \mathrm{s}$ \\
\hline$T_{L 2 C A P h}$ & L2CAP header & 4 & $32 \mu \mathrm{s}$ \\
\hline$T_{o p}$ & ATT opcode & 1 & $8 \mu \mathrm{s}$ \\
\hline
\end{tabular}




\begin{tabular}{|c|c|c|c|}
\hline$T_{A T T h}$ & Attribute handle & 2 & $16 \mu \mathrm{s}$ \\
\hline$T_{p a y}$ & Application payload & $0-20$ & $0-160 \mu \mathrm{s}$ \\
\hline$T_{C R C}$ & CRC & 3 & $24 \mu \mathrm{s}$ \\
\hline
\end{tabular}

Equations (2) and (3) represent the transmission times for the master $\left(T_{m}\right)$ and slave $\left(T_{s}\right)$ packets, respectively.

$$
\begin{gathered}
T_{m}=T_{p r e}+T_{a a}+T_{l h}+T_{C R C} \\
T_{s}=T_{p r e}+T_{a a}+T_{l h}+T_{C R C}+T_{p a y}+T_{A T T h}+T_{o p}+T_{L 2 C A P}
\end{gathered}
$$

We consider in this analysis that the master does not send any information to the slave; therefore, the link layer payload is empty. On the other hand, the slave sends a packet with the maximum application payload size (20 bytes in the ATT value field) to the master. According to equations (2) and (3), $T_{m}$ is equal to $80 \mu \mathrm{s}$ and $T_{s}$ is $296 \mu \mathrm{s}$. Given that the guard time between packets is $T_{I F S}=150 \mu \mathrm{s}$, the total time for the exchange of packets between the master and the slave $\left(T_{m s}\right)$, including the guard times, is equal to $676 \mu \mathrm{s}$ in this case, as given by Equation (4).

$$
T_{m s}=T_{m}+T_{I F S}+T_{s}+T_{I F S}
$$

Therefore, if the BLE modules could transmit continuously, it would be possible to transmit 1479 packets per second on each direction. Taking into account the maximum payload size ( 20 bytes), we can conclude that the maximum goodput (throughput at the application layer) provided by a BLE network would be equal to $236.7 \mathrm{kbps}$.

Given that data transmission via BLE is not continuous, the actual goodput of a BLE connection $\left(S_{a p p}\right)$ is expressed, instead, by Equation (5), where $N_{\text {not }}$ is the number of notifications per connection event, $L_{\text {data }}$ is the payload length at application level, in bits, and $T_{e c i}$ is the effective connection interval.

$$
S_{a p p}=\frac{N_{\text {not }} L_{\text {data }}}{T_{e c i}}
$$

Fig. 3 illustrates the maximum theoretical goodput as a function of $T_{e c i}$, for different values of $N_{\text {not }}$, using $L_{\text {data }}=20$ bytes. 


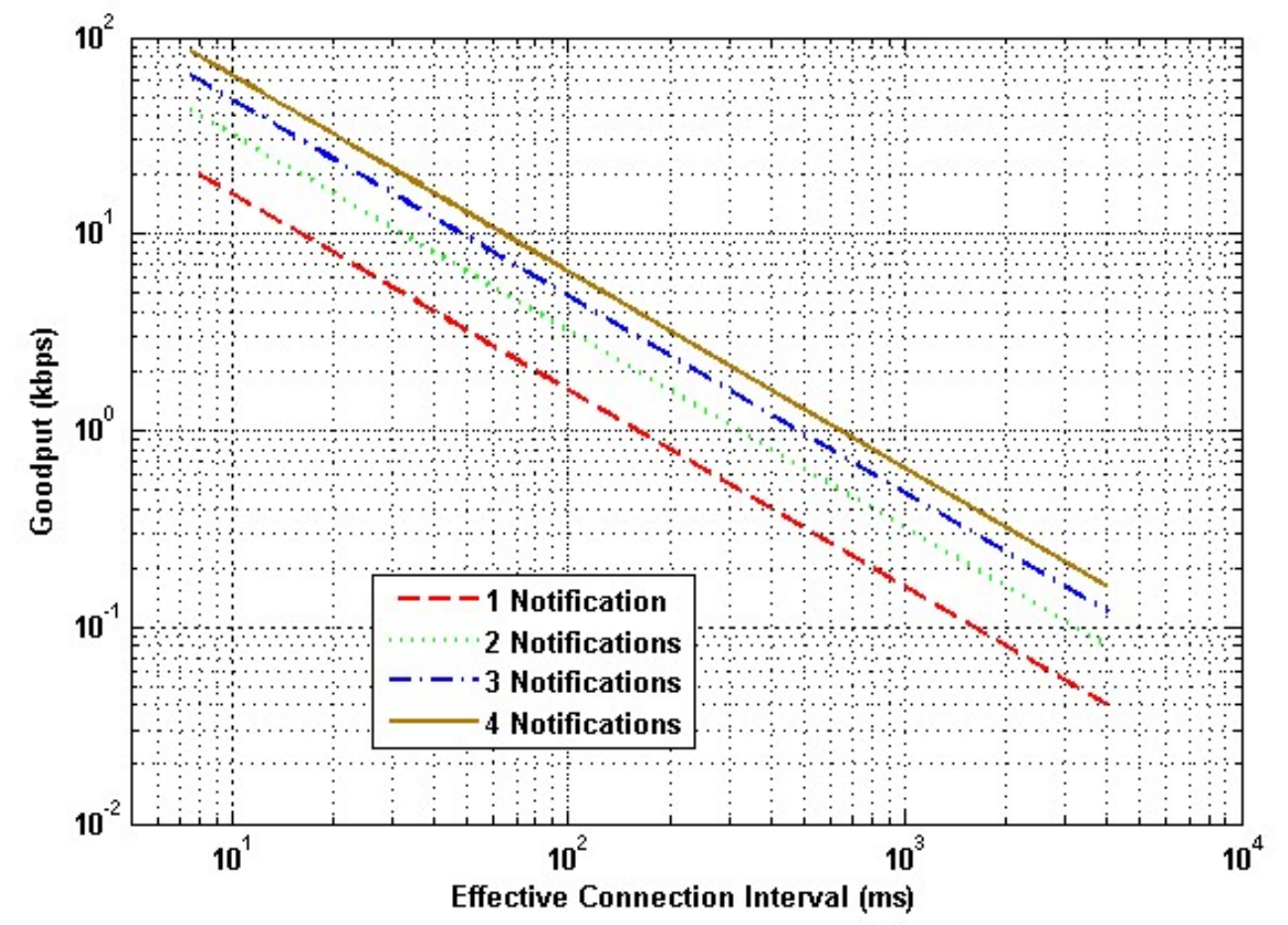

Fig. 3 Maximum BLE goodput in function of $T_{E C I}$ and the number of notifications

Considering the minimum allowed value for $T_{e c i}(7.5 \mathrm{~ms})$, for example, the goodput with a single notification is $21.3 \mathrm{kbps}$. It would be necessary to have 11 notifications per connection event in order to achieve approximately the same goodput obtained in the scenario of continuous transmission $(236.7 \mathrm{kbps})$. However, in practice, it was not possible to achieve this goodput with the tested BLE devices due to implementation constraints, which limit both the minimum reliable connection interval and the maximum number of notifications per connection event, as the results presented in section 4 show.

\subsection{Energy Consumption}

During a connection event, the BLE transceiver on the slave device switches among several states, which includes pre-processing, reception, transmission and post processing states. Using equation (6) we can calculate the average current $\left(I_{o n}\right)$ during the time that the device remains awake $\left(T_{o n}\right)$ in a connection event, based on the dwelling time in each state $\left(T_{s_{i}}\right)$ and the corresponding current consumption $\left(I_{s_{i}}\right)$, where $i$ is the index of the state and $N_{s}$ is the number of states. 


$$
I_{\text {on }}=\frac{\sum_{i=1}^{N_{S}}\left(T_{S_{i}} I_{S_{i}}\right)}{T_{o n}}
$$

In order to calculate the overall average current $\left(I_{a v g}\right)$, we have to take into account the time that the device spends in the sleep mode during $T_{e c i}$, as well as the respective current consumption $\left(I_{\text {sleep }}\right)$, as shown in equation (7).

$$
I_{a v g}=\frac{I_{o n} T_{o n}+I_{s l e e p}\left(T_{e c i}-T_{o n}\right)}{T_{e c i}}
$$

In order to estimate the battery lifetime $\left(T_{b a t}\right)$, in hours, we use the value obtained in (7) and the battery capacity $\left(C_{b a t}\right)$, in $\mathrm{mAh}$, as shown in equation (8).

$$
T_{b a t}=\frac{C_{b a t}}{I_{a v g}}
$$

Results concerning the energy consumption and battery lifetime of the sensor nodes are presented in section 4.4 .

\subsection{Number of Supported Slaves}

This section evaluates the number of slaves that a BLE network can support as a function of $T_{e c i}$. Unlike other works, this paper consider that the amount of data generated for transmission per unit of time is constant, which means that the amount of data that needs to be transmitted per connection event is proportional to $T_{e c i}$.

This paper uses traffic parameters from a real application of a wearable wireless posture monitoring system [20]; nevertheless, this analysis is generalized for systems with other parameter values in terms of number of sensors per node, sampling resolution and sampling frequency.

Each sensor node (BLE slave) in this system contains three sensors (accelerometer, magnetometer and gyroscope), each one with three axes, for a total of nine sensor values. These values are sampled at 16 bits; therefore, the amount of data generated for each sample point is 144 bits, or 18 bytes. Since this value is close to the maximum payload length allowed by BLE ( 20 bytes), we consider in this analysis a payload length $\left(L_{\text {data }}\right)$ of 144 bits, which means that, in this case, each notification packet carries data from a single sample point. This system is configured to operate with a frame rate of 
$30 \mathrm{fps}$, which is a typical value for motion capture applications. This value corresponds to a sampling frequency $\left(f_{s}\right)$ of $30 \mathrm{~Hz}$.

Table 2 summarizes the relevant parameters of a sensor node, as well as the values associated to the considered posture monitoring application.

Table 2 Relevant parameters and values for the considered BAN application

\begin{tabular}{|c|c|c|}
\hline Parameter & Name & Value \\
\hline Number of sensors per node & $N_{s}$ & 9 \\
\hline Sampling resolution & $Q_{s}$ & $16 \mathrm{bits}$ \\
\hline Sampling frequency & $f_{s}$ & $30 \mathrm{~Hz}$ \\
\hline Payload length & $L_{\text {data }}$ & $144 \mathrm{bits}$ \\
\hline
\end{tabular}

The effective connection interval $\left(T_{e c i}\right)$ depends on the number of notifications per connection event $\left(N_{n o t}\right)$, the payload length $\left(L_{\text {data }}\right)$, the number of sensors $\left(N_{s}\right)$, the sampling resolution $\left(Q_{s}\right)$ and the sampling frequency $\left(f_{s}\right)$, as shown in equation (9).

$$
T_{e c i}=\frac{N_{\text {not }} L_{\text {data }}}{N_{s} Q_{s} f_{s}}
$$

As equation (10) shows, the number of supported slaves $\left(N_{\text {slaves }}\right)$ can be obtained through the division of $T_{e c i}$ by the time $\left(T_{\text {total }}\right)$ required for a slave to transmit all its notifications $\left(N_{n o t}\right)$ in a connection event. This time, in turn, is the product of $N_{n o t}$ by the time required for a master-slave packet exchange $\left(T_{m s}\right)$. Replacing $T_{e c i}$ by the variables in the right-hand side of equation (9) and simplifying, we reach the conclusion that $N_{\text {slaves }}$ is proportional to $L_{\text {data }}$ and inversely proportional to the product of $N_{s}, Q_{s}, f_{s}$ and $T_{m s}$. Therefore, it is independent of $T_{e c i}$.

$$
N_{\text {slaves }}=\frac{T_{e c i}}{T_{\text {total }}}=\frac{T_{e c i}}{N_{\text {not }} T_{m s}}=\frac{L_{\text {data }}}{N_{s} Q_{s} f_{s} T_{m s}}
$$

\section{4 eLPRT Protocol}

The eLPRT protocol is an enhanced version of the LPRT (Low Power Real Time) protocol. In comparison with the MAC protocols defined by the IEEE 802.15.4 
standard, it introduces several mechanisms designed to increase the reliability against errors, improve the bandwidth utilization and increase the number of supported devices. The eLPRT protocol defines a superframe structure composed of a beacon, transmitted by the base station, a contention access period (CAP), where reservation requests can be made, and a contention-free period (CFP), where the allocations for the nodes' transmissions are reserved.

The eLPRT protocol divides each superframe into a fixed number of mini-slots $\left(N_{m s s}\right)$. The number of mini-slots required for the transmission of a packet from a sensor node $\left(N_{m s p}\right)$ in a superframe is given by equation (11).

$$
N_{m s p}=\left\lceil\frac{\text { Tpacket Nmssf }}{T_{s f}}\right\rceil+N_{m s g}
$$

where $T_{s f}$ is the superframe period, $N_{m s g}$ is the number of guard mini-slots allocated to the packet in the superframe and $T_{\text {packet }}$ is the packet transmission time, which depends on the packet length $\left(L_{\text {packet }}\right)$ and the bit rate available at the physical layer $\left(R_{e L P R T}\right)$ for the eLPRT protocol:

$$
T_{\text {packet }}=\frac{L_{\text {packet }}}{R_{\text {eLPRT }}}
$$

The number of supported sensor nodes $\left(N_{\text {nodes }}\right)$ is given by equation (13), where $N_{m s C F P}$ is the number of mini-slots in the CFP.

$$
N_{\text {nodes }}=\frac{N_{m s C F P}}{N_{m s p}}
$$

The number of mini-slots in the CFP, in turn, can be calculated using equation (14), where $T_{C F P}$ is the duration of the CFP.

$$
N_{m s C F P}=\frac{N_{m s s f} T_{C F P}}{T_{s f}}
$$

For a given superframe period, the maximum number of supported nodes is achieved when the minimum duration $\left(T_{C A P \min }\right)$ of the CAP is used. 


\section{Results and Discussion}

The experimental tests described in this section were made using two different platforms: Texas Instruments CC2540 modules, with BLE stack version 1.3.2, and a Nexus 5 smartphone, with Android 4.4. CC2540 modules were used as slaves in the tested BLE piconets, whereas both platforms were used as the master device in different tests.

During the experimental tests, we took care to assure that the tested networks were free from significant interference from other sources, such as nearby Wi-Fi networks, through the use of an Wi-Spy 2.4x spectrum analyzer, in order to avoid the occurrence of unaccounted packet errors.

\subsection{Notifications per Connection Event}

The BLE specification does not impose limits on the number of notifications per connection event that a slave can send. However, tests performed have shown that, using the maximum application payload allowed for a BLE notification (20 bytes), a CC2540 module in a slave role can only send up to three notifications per connection event. It was also noticed that it is possible to send more notifications per connection event when smaller payload lengths are used, as shown in Table 3. This limitation can be attributed to constraints of the BLE implementation on these modules.

Table 3 Correspondence between the payload size and the maximum number of notifications

\begin{tabular}{|c|c|}
\hline Payload length (bytes) & Number of notifications \\
\hline 17 to 20 & 3 \\
\hline 13 to 16 & 4 \\
\hline 10 & 5 \\
\hline
\end{tabular}

\subsection{Packet Losses}

For the first experimental test of this section, we used the maximum payload possible for a notification ( 20 bytes) and measured the packet loss rate (PLR), after the 
transmission of 1000 notifications, for various values of the connection interval, keeping the slave latency fixed in zero. We used one CC2540 module as slave and tested the two different platforms as the master device.

Fig. 4 presents the PLR results with three notifications per connection event. For very low values of connection interval, the PLR is very high (close to 100\%) in both cases. The PLR decreases as the connection interval increases, but packet losses are no longer observed only for connection intervals higher than $25 \mathrm{~ms}$, for the CC2540 module, and higher than $60 \mathrm{~ms}$, for the Android smartphone.

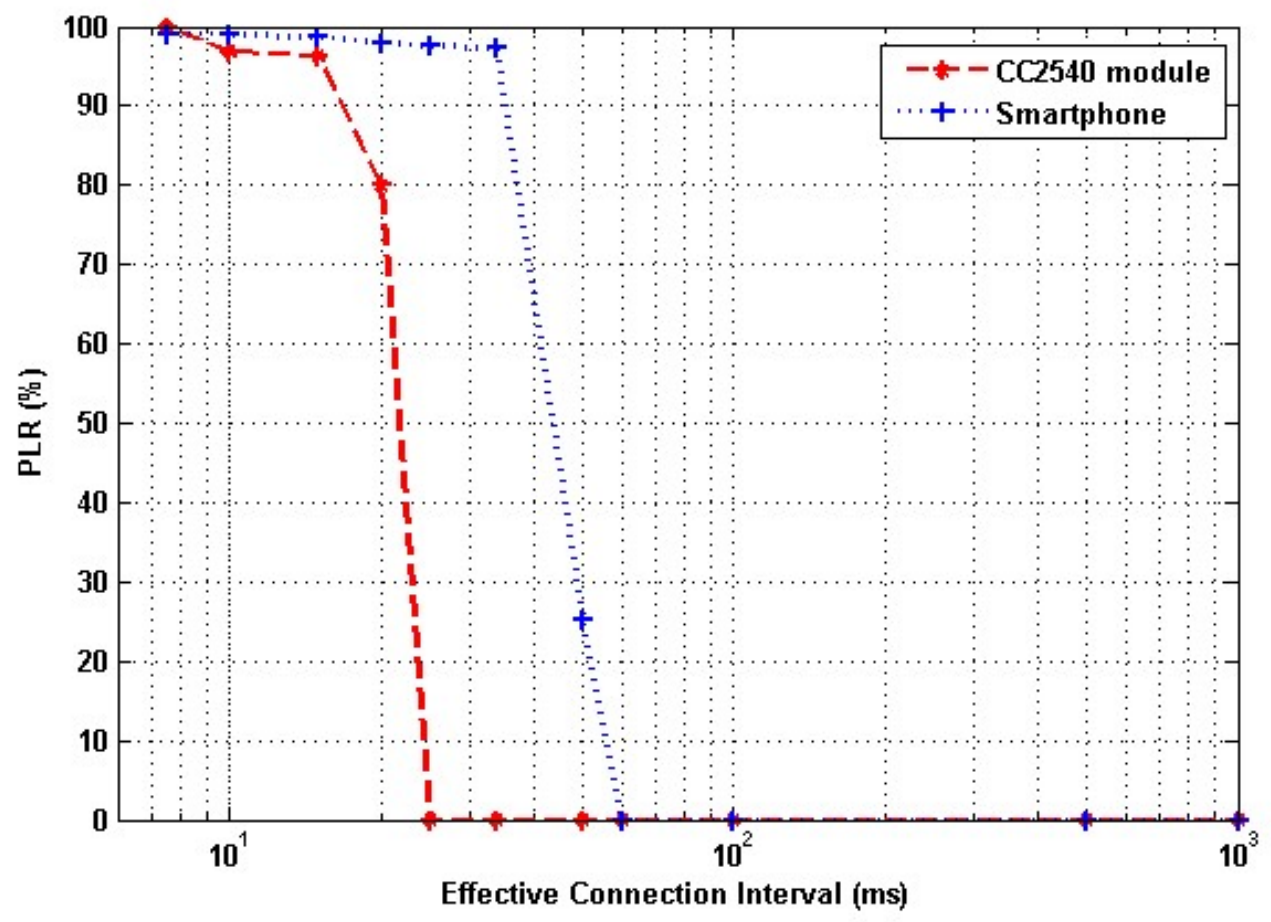

Fig. 4 PLR results with three notifications when the master is either a CC2540 module or a smartphone

For the second test, the number of notifications per connection event was varied from one to three. This test was performed using the smartphone as master, with connection intervals near the range in which packet losses were noticed in the previous test. Through Fig. 5, it is possible observe that, as the number of notifications per connection event decreases, the minimum connection interval with error-free transmissions also decreases. As the figure shows, for three, two and one notification, the minimum connection interval without packet losses is, respectively, $60 \mathrm{~ms}, 50 \mathrm{~ms}$ and $20 \mathrm{~ms}$. 


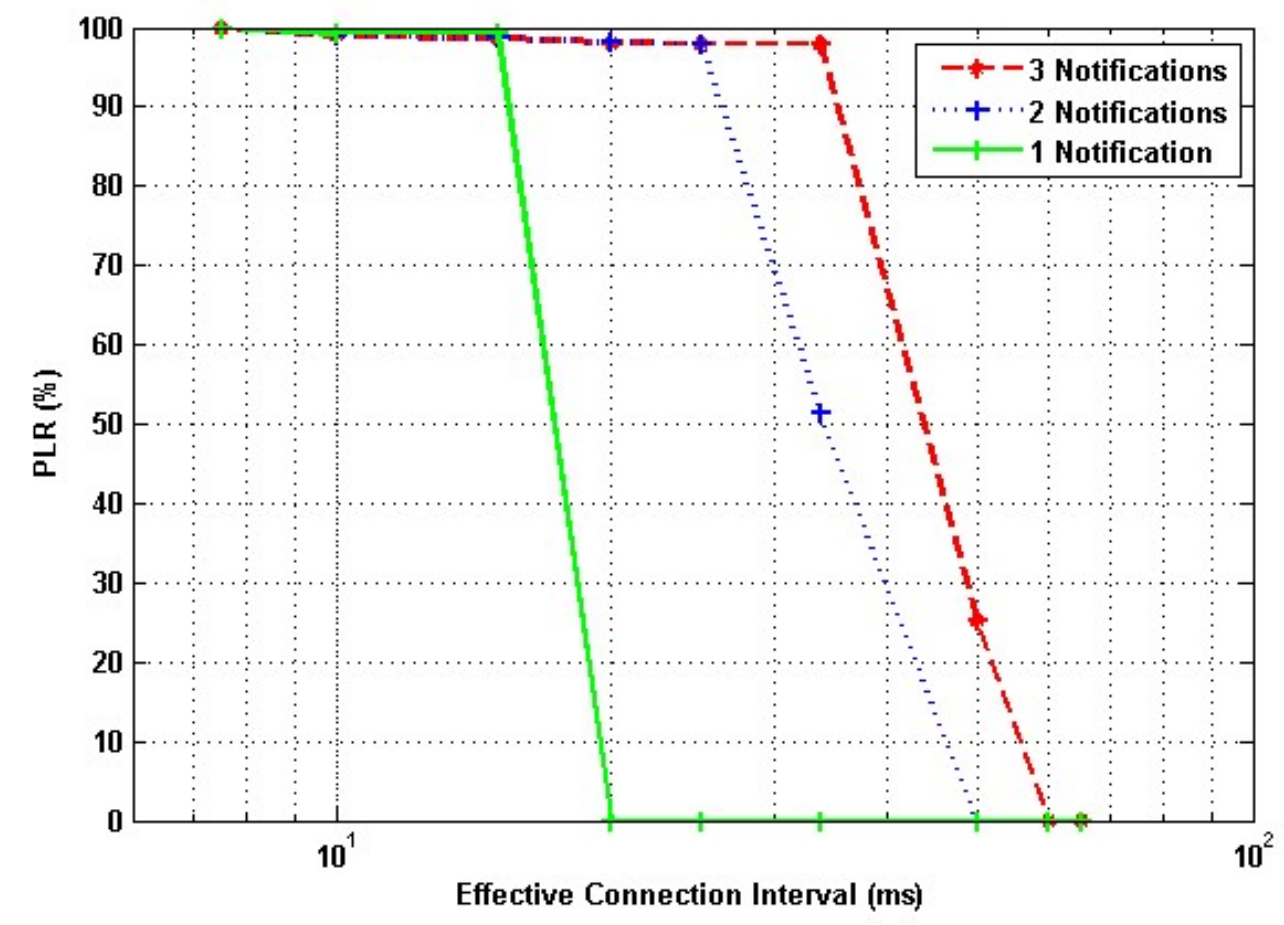

Fig. 5 PLR values with variable number of notifications with the smartphone as master

For both tests, the packet losses are due to implementation constraints in terms of hardware and/or software on the used devices, since the BLE specification defines that the connection interval can be as low as $7.5 \mathrm{~ms}$.

\subsection{Support of Multiple Slaves}

In order to validate experimentally the operation of the BLE protocol with multiple simultaneously active slaves, a network with one master (Nexus 5 smartphone) and four slaves (CC2540 modules) was set up. The connection between the master and one of the slaves was monitored for a period of 18 consecutive hours. The connection interval was set to $100 \mathrm{~ms}$, the number of notifications per connection event was set to three, and the payload length was 20 bytes.

During the whole duration of the test, the delivery ratio of the connection was $100 \%$. Therefore, it can be concluded that the BLE protocol is able to avoid packet losses due to the overlapping between the transmissions from different slaves, unlike other protocols, such as the unslotted CSMA/CA protocol of the IEEE 802.15.4, which is vulnerable to collisions due to clock-drift effects [25]. 


\subsubsection{Maximum Number of Slaves}

This section provides a comparison of the number of supported sensor nodes between the BLE and eLPRT protocols, using the traffic parameters of the wireless posture monitoring application described in section 3.3.

As an example of the evaluation of the number of supported slaves for the BLE protocol, consider the parameter values presented in Table 2. According to equation (9), we have $T_{e c i}=33.3 \mathrm{~ms}$ for $N_{\text {not }}=1$, and $T_{e c i}=100 \mathrm{~ms}$ for $N_{\text {not }}=3$. According to equations (2) and (3), the transmission time $\left(T_{m}\right)$ for a master packet with empty payload is $80 \mu \mathrm{s}$, whereas the transmission time $\left(T_{s}\right)$ of a slave packet containing 18 bytes of payload is $280 \mu \mathrm{s}$. Therefore, the time necessary to exchange packets between the master and the slave $\left(T_{m s}\right)$, including the guard times, is equal to $660 \mu \mathrm{s}$. The total time $\left(T_{\text {total }}\right)$ required is proportional to the number of notifications, which means that, with these traffic parameters, it is possible to accommodate a maximum of 50 slaves, according to equation (10), independently of the connection interval.

The analysis performed in section 3.3 can be used in other traffic scenarios. As an example, consider an ECG sensor $\left(N_{s}=1\right)$ sampled with frequency $f_{s}=250 \mathrm{~Hz}$ and resolution $Q_{s}=12$ bits. In this case, we would have $T_{e c i}=48 \mathrm{~ms}$ for $N_{\text {not }}=1$, and $T_{e c i}=144 \mathrm{~ms}$ for $N_{n o t}=3$, according to equation (9), and the maximum number of slaves would increase to 72 , according to equation (10).

Regarding the eLPRT protocol, the relevant parameters for this evaluation are summarized in Table 4 . The number of mini-slots per superframe $\left(N_{m s s f}\right)$ was set to 500 , whereas the minimum duration of the contention access period (CAP) was set to $11 \mathrm{~ms}$, which is sufficient for the exchange of two packets with maximum size, including the backoff imposed by CSMA/CA mechanism and the space between packets. Therefore, the maximum duration of the contention-free period (CFP) is given by the chosen superframe period $\left(T_{s f}\right)$ minus a fixed reserved period, with duration of $15.26 \mathrm{~ms}$, which 
is composed of the minimum CAP $\left(T_{C A P \min }\right)$ plus the time required for the transmission of a beacon of maximum size $(4.26 \mathrm{~ms})$.

Table 4 eLPRT protocol parameters

\begin{tabular}{|c|c|c|}
\hline Parameter & Name & Value \\
\hline Bit rate & $R_{e L P R T}$ & $250 \mathrm{kbps}$ \\
\hline PHY layer overhead & $L_{o P H Y}$ & 6 bytes \\
\hline MAC layer overhead & $L_{o M A C}$ & 9 bytes \\
\hline Payload length & $L_{P a y}$ & variable \\
\hline Superframe period & $T_{s f}$ & variable \\
\hline Mini-slots per superframe & $N_{m s s f}$ & 500 \\
\hline Minimum CAP size & $T_{C A P m i n}$ & $11 \mathrm{~ms}$ \\
\hline Maximum beacon duration & $T_{b m a x}$ & $4.256 \mathrm{~ms}$ \\
\hline
\end{tabular}

When $T_{s f}=100 \mathrm{~ms}$, for example, the maximum duration of the CFP is $T_{C F P}=84.74$ ms, which corresponds to $N_{m s C F P}=423$ mini-slots, according to equation (14). The payload length $\left(L_{p a y}\right)$, in bits, is the product of the number of sensors per node $\left(N_{s}\right)$, the sampling resolution $(Q s)$, the sampling frequency $\left(f_{s}\right)$ and the superframe period $\left(T_{s f}\right)$, as shown in Equation (15).

$$
L_{\text {pay }}=N_{s} Q_{s} f_{s} T_{s f}
$$

Using the parameter values provided in Table 2, the payload length in this case is 54 bytes, which means that the total packet length, including the protocol overhead (at PHY and MAC layers) is 69 bytes. Given that the bit rate for the eLPRT protocol is 250 $\mathrm{kbps}$, the packet transmission time ( $\left.T_{\text {packet }}\right)$ is equal to $2.2 \mathrm{~ms}$, which requires at least 11 mini-slots. An additional mini-slot is used in order to provide a guard time between the transmissions of consecutive sensor nodes $\left(N_{m s g}=1\right)$, thus resulting in 12 mini-slots for each sensor node, according to equation (11). Through the division of the number of mini-slots available in the CFP $\left(N_{m s C F P}=423\right)$ by the number of mini-slots required per sensor node packet $\left(N_{m s p}=12\right)$, according to equation (13), we can reach the conclusion 
that the maximum number of sensor nodes supported by the eLPRT protocol, considering this superframe period and traffic parameters, is $N_{\text {nodes }}=35$.

Results concerning the number of supported sensor nodes for other values of the transmission interval ( $T_{e c i}$ for BLE and $T_{s f}$ for eLPRT) are presented in Fig. 6. For the BLE protocol, the number of supported slaves for the considered BAN application is independent of the effective connection interval $\left(T_{e c i}\right)$, as concluded before. This happens because the sensor data is sent in separate packets (notifications) of fixed payload length, and the protocol overhead associated with each packet is also fixed.

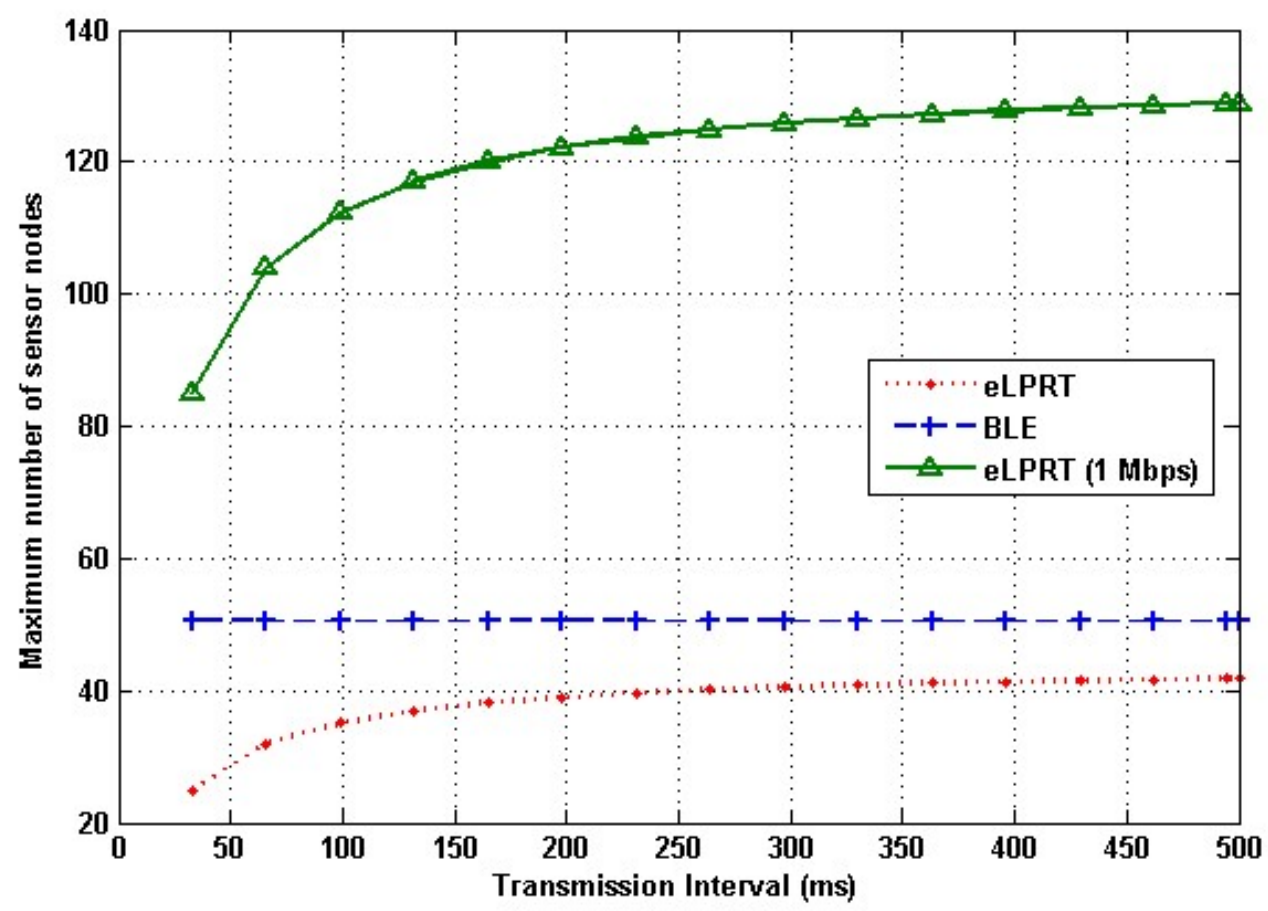

Fig. 6 Number of supported slaves in the traffic of the considered BAN application for the BLE and eLPRT protocols

On the other hand, in the case of eLPRT protocol, as the superframe period $\left(T_{s f}\right)$ increases, the number of supported sensor nodes increases as well. This happens because the payload length $\left(L_{p a y}\right)$ also increases, whereas the length of the protocol overhead ( $L_{O P H Y}$ and $\left.L_{O M A C}\right)$ does not change, which means that its influence decreases, in relative terms. In both cases, the larger the transmission interval, the higher becomes the delay suffered by the first collected sample points. 
In order to provide a comparison of the efficiency of these protocols under similar physical layer conditions, we also present in Fig. 6 an estimate of the number of supported nodes for the eLPRT protocol if the bit rate was $1 \mathrm{Mbps}$ (which corresponds to the bit rate of the BLE protocol) instead of $250 \mathrm{kbps}$. In this case, the number of nodes supported by the eLPRT protocol would be higher than for the BLE protocol over all range of transmission intervals.

\subsubsection{Platform support}

We performed experimental tests in order to evaluate the support provided by the tested BLE platforms to networks with multiple slaves. As before, we used a CC2540 module as slave (peripheral station), and either a CC2540 module or a Nexus 5 Android smartphone as master (central station).

With a CC2540 module as master and $T_{e c i}=100 \mathrm{~ms}$, it was only possible to transmit all three notifications per connection event required by the considered BAN application with a single slave in the network. When more than one slave was connected to the network (either two or three slaves), it was observed that the master received only one out of the three notifications per connection event, verifying the loss of the other two packets. This result is in accordance with the documentation of the BLE protocol stack used in this paper [26].

Therefore, in order to circumvent this limitation while maintaining a sampling frequency of $30 \mathrm{~Hz}$, as required, the connection interval would have to be set to a value close to $33.3 \mathrm{~ms}$ (the closest value allowed by BLE is $33.75 \mathrm{~ms}$ ). With this value and one notification per connection event, the network operated without problems with up to the three slaves, which is the maximum number of simultaneous connections supported by the central station in this version of the BLE stack [26].

When the Nexus 5 smartphone was used as the central station, the limitations referred above were not observed. In tests with up to four slaves using $T_{e c i}=100 \mathrm{~ms}$ and three notifications per connection event, all packets were successfully transmitted. 


\subsection{Energy Consumption}

During a connection event, the BLE transceiver switches among different states. In [27], the authors used CC2540 modules to measure the dwelling time in each state and the corresponding current consumption for a slave device. In this section, we use these values, which are shown in Table 5, to calculate the energy consumption of a BLE slave. The Rx and Tx times presented in this table correspond to the values of $T_{m}$ and $T_{s}$, respectively, which were calculated using equations (2) and (3). As before, we consider an empty payload for the master and a payload length of 20 bytes for the slave. Table 3 illustrates the sequence of states associated to sending just one notification from slave to master. In practice, there will be as many passages by the Rx, Rx-to-Tx and Tx states as the number of notifications per connection event. When two or more notifications are used, the Tx-to-Rx state, which has the same duration and current consumption as the Tx-to-Rx state, is also present.

Table 5 Dwelling time and current consumption for the different BLE transceiver states during a connection event

\begin{tabular}{|c|c|c|c|}
\hline State & Description & Time $(\boldsymbol{\mu s})$ & Current (mA) \\
\hline $\mathbf{1}$ & Wake-up & 400 & 6.0 \\
\hline $\mathbf{2}$ & Pre-processing & 340 & 7.4 \\
\hline $\mathbf{3}$ & Pre-Rx & 80 & 11.0 \\
\hline $\mathbf{4}$ & $\mathrm{Rx}$ & 80 & 22.1 \\
\hline $\mathbf{5}$ & Rx-to-Tx & 150 & 7.4 \\
\hline $\mathbf{6}$ & Tx & 296 & 17.5 \\
\hline $\mathbf{7}$ & Post-processing & 1280 & 7.4 \\
\hline $\mathbf{8}$ & Pre-sleep & 160 & 4.1 \\
\hline
\end{tabular}

As an example, if we consider a single notification with sleep current $\left(I_{\text {sleep }}\right)$ of $1 \mu \mathrm{A}$ and $T_{e c i}=100 \mathrm{~ms}$, the average slave current $\left(I_{\text {avg }}\right)$, calculated using equation (7), is equal to $0.237 \mathrm{~mA}$. Under these conditions, assuming the use of a battery with capacity $\left(C_{b a t}\right)$ of $120 \mathrm{mAh}$, such as the LIR2450 lithium-ion rechargeable coin cell battery, the 
estimated battery lifetime $\left(T_{b a t}\right)$, using equation (8) would be 506.3 hours, or approximately 21 days. Notice that these results take into account only the consumption of the CC2540 module, not including the consumption of other components that may be required by the application, such as the sensors.

Fig. 7 illustrates the battery lifetime as a function of the effective connection interval, considering one, two or three notifications per connection event. In this scenario, as $T_{e c i}$ increases, the battery lifetime also increases, because the sleep period increases, whereas the active period remains the same.

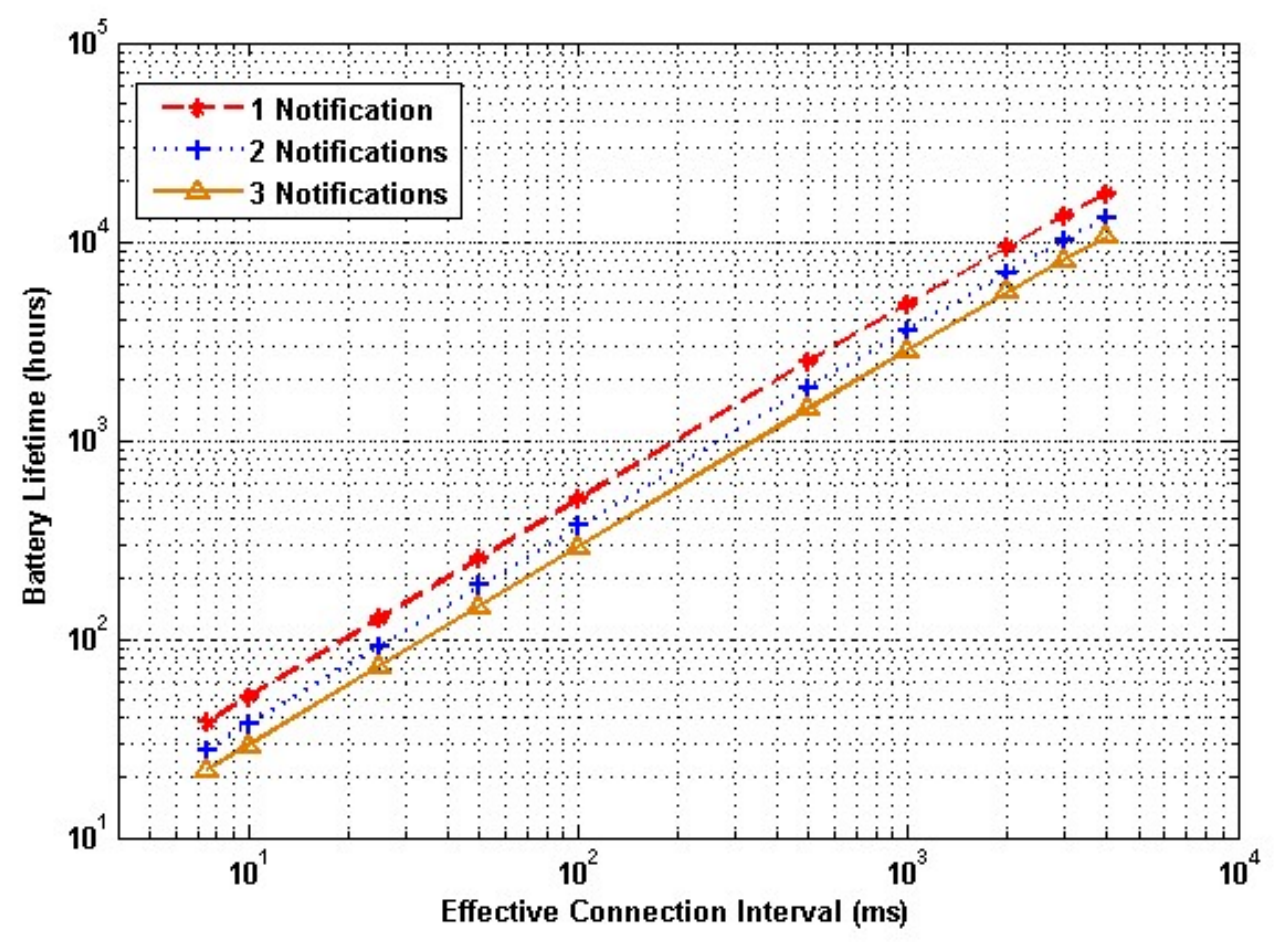

Fig. 7 Battery lifetime as a function of the effective connection interval and the number of notifications

\subsubsection{BLE vs. eLPRT}

This section provides a comparison of the current consumption of the BLE protocol, using a CC2540 module, and the eLPRT protocol, using a CC2530 module, for the scenario of the wireless posture monitoring application, with sampling frequency $f_{s}=30 \mathrm{~Hz}$ and data length of 18 bytes per sample point. Therefore, unlike the previous results, in this case the amount of data to be transmitted is proportional to the 
transmission interval, which corresponds to the effective connection interval, for the BLE protocol, and to the superframe period, for the eLPRT protocol.

This comparison is based on the current consumption of the sensor node at each state, as well as the respective dwelling time, associated to each of the transceivers. For the BLE transceiver, these values were obtained from [27] and are presented in Table 5. The times for the Rx and Tx states were adjusted taking into account the payload length of 18 bytes instead of 20 bytes, and the Tx power was set to $4 \mathrm{dBm}$, which means that the Tx current was increased to $31.6 \mathrm{~mA}$.

For the eLPRT transceiver, we performed experimental measurements in order to obtain the required values. The Tx power for the CC2530 modules was set to $4.5 \mathrm{dBm}$, which is similar to the value used for the CC2540 modules, in order to provide a fair comparison between the two platforms.

In order to characterize the consumption of a sensor node using the eLPRT protocol, we measured its current consumption and dwelling time at each state during the active periods, which correspond to the transmission of data and reception of the beacon. Table 6 presents the values concerning the transmission of the collected sensor data from the sensor node to the base station, whereas Table 7 concerns the values measured for the reception (by the sensor node) of the beacon sent by the base station. These measurements were made using a superframe period of $100 \mathrm{~ms}$. In this case, the length of the data packet is 69 bytes, where 54 bytes correspond to the payload (data from three sample points) and 15 bytes are from the protocol overhead (headers and trailers). For other values of the superframe period, the dwelling time in the Tx state was adjusted accordingly, taking into account the number of sample points. 
Table 6 Dwelling time and current consumption for the eLPRT transceiver states during a data packet transmission

\begin{tabular}{|c|c|c|c|}
\hline State & Description & Time $(\boldsymbol{\mu s})$ & Current $(\mathbf{m A})$ \\
\hline $\mathbf{1}$ & Wake-up & 100 & 7.4 \\
\hline $\mathbf{2}$ & Rx idle & 500 & 24.3 \\
\hline $\mathbf{3}$ & Rx-to-Tx & 100 & 13.3 \\
\hline $\mathbf{4}$ & Tx & 2500 & 33.5 \\
\hline $\mathbf{5}$ & Pre-sleep & 100 & 15.0 \\
\hline
\end{tabular}

Table 7 Dwelling time and current consumption for the eLPRT transceiver states during a beacon reception

\begin{tabular}{|c|c|c|c|}
\hline State & Description & Time $(\boldsymbol{\mu s})$ & Current $(\mathbf{m A})$ \\
\hline $\mathbf{1}$ & Wake-up & 100 & 7.4 \\
\hline $\mathbf{2}$ & Rx idle & 1200 & 26.8 \\
\hline $\mathbf{3}$ & Rx active & 700 & 24.8 \\
\hline $\mathbf{4}$ & Rx idle and post-processing & 1500 & 26.9 \\
\hline $\mathbf{5}$ & Pre-sleep & 500 & 7.6 \\
\hline
\end{tabular}

Fig. 8 presents the average current consumption as a function of the transmission interval for both protocols. According to these results, it is possible to see that the current consumption decreases, for both protocols, as the transmission interval increases. The decrease in the BLE protocol is due to the reduction of the relative weight of the accessory states (wake-up, pre-processing, pre-Rx, post-processing and presleep), which appear only once per transmission interval, when compared to the main states ( $\mathrm{Rx}$ and $\mathrm{Tx}$ ), whose number of occurrences increases with the transmission interval. The decrease in the current consumption as the transmission interval increases is more pronounced for the eLPRT protocol, not only due to the decreased weight of the accessory states, but also due to the decreased contribution of the protocol overhead, 
since the packet header has a fixed size whereas the payload increases with the transmission interval.

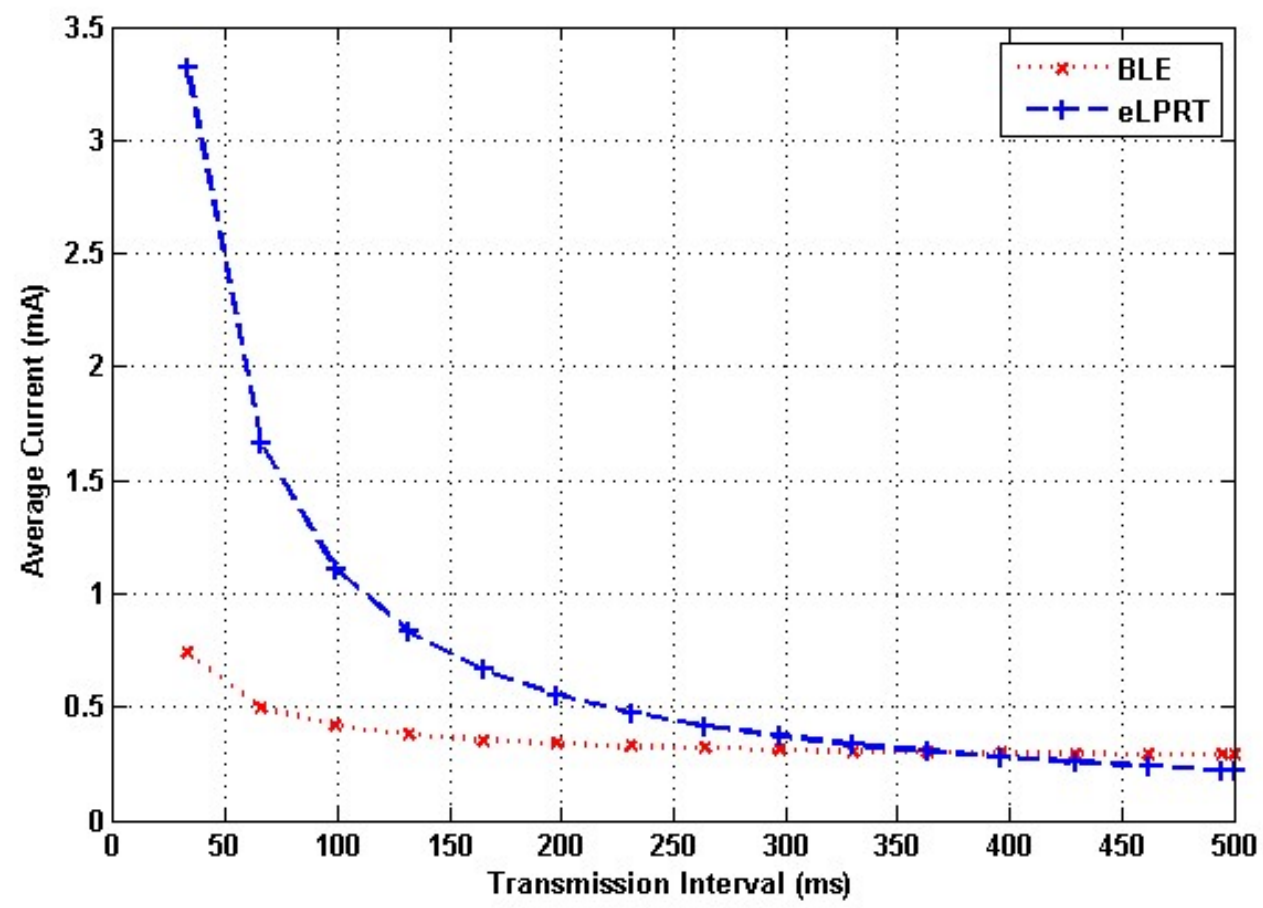

Fig 8. Current consumption comparison between BLE and eLPRT.

The BLE protocol presents lower current consumption than the eLPRT protocol for transmission intervals up to $350 \mathrm{~ms}$. Notice that, in practice, it would not be possible to use a superframe period higher than $200 \mathrm{~ms}$ for the eLPRT protocol, because of the limit of 133 bytes (at the physical layer) imposed by the IEEE 802.15 .4 standard for the maximum size of a packet. Likewise, it would not be possible to use $T_{e c i}$ higher than 100 ms with the CC2540 modules, due to the implementation constraints referred before, which prevent the use of more than three notifications per connection event

\section{Conclusion}

This work presented a performance evaluation of the BLE protocol, and a comparison with the eLPRT MAC protocol, which was implemented over the IEEE 802.15.4 physical layer. Unlike other works, this paper considered the transport of the 
traffic of multiple high data rate nodes, in traffic scenarios where the amount of data generated by the sensor nodes is proportional to the transmission interval.

For these traffic scenarios, results have shown that, in the case of the eLPRT protocol, there is a tradeoff between delay, on the one hand, and the number of supported nodes and energy consumption, on the other hand. An increase of the superframe period increases the number of supported nodes and reduces the energy consumption, because the payload increases, reducing the overhead of the eLPRT protocol. However, the delay associated to the transmission interval also tends to increase. In contrast, for the BLE protocol, the tradeoff was verified only between the delay and the energy consumption, whereas the number of supported nodes was independent of the connection interval.

This work presented a detailed experimental evaluation of two different BLE platforms: CC2540 modules and a Nexus 5 smartphone. Results have shown the occurrence of packet losses for small values of connection interval with both platforms. The CC2540 modules also presented other implementation constraints: limits on the number of notifications per connection event and the number of slaves per network, as well as the restriction of a single notification in networks with multiple slaves. These results indicate that the requirements of high data rate sensor nodes were overlooked in the design and implementation of the tested platforms.

Nevertheless, the BLE protocol presented encouraging results. Unlike the IEEE 802.15.4 protocol, the BLE performance is not affected by collisions, which is particularly important in scenarios with multiple sensor nodes and high traffic load, such as some BAN applications. Moreover, BLE presented lower energy consumption and the capacity to support more sensor nodes than the IEEE 802.15.4-based eLPRT protocol with the same traffic parameters, in part due to the higher bit rate $(1 \mathrm{Mbps}$ compared to $250 \mathrm{kbps}$ ). The fact that the BLE interface tends to be natively integrated in most smartphones is another advantage over wireless technologies such as ZigBee. Although ZigBee may be integrated in a mobile device using, for example, a microSD card, this solution increases the cost and is less practical. 


\section{Acknowledgments}

This work has been supported by FCT (Fundação para a Ciência e Tecnologia) in the scope of the projects UID/EEA/04436/2013 and UID/CTM/50025/2013, and by FEDER funds through the COMPETE 2020 Programme.

\section{References}

1. Patel, M., \& Wang, J. (2010). Applications, challenges, and prospective in emerging body area networking technologies. Wireless Communications, IEEE, 17(1), 80-88.

2. Pantelopoulos, A., \& Bourbakis, N. G. (2010). A survey on wearable sensor-based systems for health monitoring and prognosis. Systems, Man, and Cybernetics, Part C: Applications and Reviews, IEEE Transactions on, 40(1), 1-12.

3. Chen, M., Gonzalez, S., Vasilakos, A., Cao, H., \& Leung, V. C. (2011). Body area networks: A survey. Mobile networks and applications, 16(2), 171-193.

4. Fernández-López, H., Afonso, J. A., Correia, J. H., \& Simoes, R. (2012). Towards the design of efficient nonbeacon-enabled ZigBee networks. Computer Networks, 56(11), 2714-2725.

5. IEEE Std 802.15.4(2011). IEEE standard for local and metropolitan area networks - Part 15.4: low-rate wireless personal area networks (LR-WPANs).

6. Gislason, D. (2008). ZigBee Wireless Networking. Newnes.

7. IEEE Std 802.15.6 (2012). IEEE standard for local and metropolitan area networks - Part 15.6: wireless body area networks.

8. Omre, A. H., \& Keeping, S. (2010). Bluetooth low energy: wireless connectivity for medical monitoring. Journal of diabetes science and technology, 4(2), 457-463.

9. Jara, A. J., Fernandez, D., Lopez, P., Zamora, M. A., Gómez-Skarmeta, A. F., \& Marin, L. (2013). Evaluation of Bluetooth Low Energy Capabilities for Tele-mobile Monitoring in Home-care. J. UCS, 19(9), 1219-1241.

10. Chan, A. M., Selvaraj, N., Ferdosi, N., \& Narasimhan, R. (2013, July). Wireless patch sensor for remote monitoring of heart rate, respiration, activity, and falls. In Engineering in Medicine and Biology Society (EMBC), 2013 35th Annual 
International Conference of the IEEE (pp. 6115-6118). IEEE.

11. Maio, A., Afonso, J. A. (2015). Wireless Cycling Posture Monitoring Based on Smartphones and Bluetooth Low Energy. World Congress on Engineering, London, United Kingdom.

12. Lin, J. R., Talty, T., \& Tonguz, O. K. (2015). On the potential of Bluetooth Low Energy technology for vehicular applications. Communications Magazine, IEEE, 53(1), 267-275.

13. Want, R., Schilit, B. N., \& Jenson, S. (2015). Enabling the Internet of Things. Computer, IEEE, 48(1), 28-35.

14. Bandyopadhyay, D., \& Sen, J. (2011). Internet of things: Applications and challenges in technology and standardization. Wireless Personal Communications, 58(1), 49-69.

15. Nieminen, J., Gomez, C., Isomaki, M., Savolainen, T., Patil, B., Shelby, Z., et al. (2014). Networking solutions for connecting Bluetooth Low Energy enabled machines to the Internet of Things. Network, IEEE, 28(6), 83-90.

16. Siekkinen, M., Hiienkari, M., Nurminen, J. K., \& Nieminen, J. (2012, April). How low energy is Bluetooth Low Energy? Comparative measurements with ZigBee/802.15.4. In Wireless Communications and Networking Conference Workshops (WCNCW), 2012 IEEE (pp. 232-237). IEEE.

17. Gomez, C., Demirkol, I., \& Paradells, J. (2011). Modeling the maximum throughput of Bluetooth Low Energy in an error-prone link. Communications Letters, IEEE, 15(11), 1187-1189.

18. Mikhaylov, K., Plevritakis, N., \& Tervonen, J. (2013). Performance analysis and comparison of Bluetooth Low Energy with IEEE 802.15.4 and SimpliciTI. Journal of Sensor and Actuator Networks, 2(3), 589-613.

19. Gomez, C., Oller, J., \& Paradells, J. (2012). Overview and evaluation of Bluetooth Low Energy: An emerging low-power wireless technology. Sensors, 12(9), 1173411753.

20. Afonso, J. A., Correia, J. H., Silva, H. R., \& Rocha, L. A. (2008). Body kinetics monitoring system. International Patent WO/2008/018810A2.

21. Paksuniemi, M., Sorvoja, H., Alasaarela, E., \& Myllyla, R. (2006, January). Wireless sensor and data transmission needs and technologies for patient monitoring in the operating room and intensive care unit. In Engineering in 
Medicine and Biology Society, 2005. IEEE-EMBS 2005. 27th Annual International Conference of the (pp. 5182-5185). IEEE.

22. Afonso, J. A., Rocha, L. A., Silva, H. R., \& Correia, J. H. (2006, December). MAC protocol for low-power real-time wireless sensing and actuation. In Electronics, Circuits and Systems, 2006. ICECS'06. 13th IEEE International Conference on (pp. 1248-1251). IEEE.

23. Afonso, J. A., Silva, H. D., Macedo, P., \& Rocha, L. A. (2011). An enhanced reservation-based MAC protocol for IEEE 802.15. 4 networks. Sensors, 11(4), 3852-3873.

24. Bluetooth SIG (2013, December). Bluetooth Specification Version 4.1 - Master Table of Contents \& Compliance Requirements.

25. Gomes, D., \& Afonso, J. A. (2014). Improving the communication reliability of body sensor networks based on the IEEE 802.15. 4 protocol. Telemedicine and eHealth, 20(3), 261-268.

26. Texas Instruments (2013). CC2540/41 Bluetooth Low Energy Software Developer's Guide v1.3.2 (SWRU271F).

27. Texas Instruments (2012). Measuring Bluetooth Low Energy Power Consumption. Application Note ANO92. 